\title{
unc-101, a gene required for many aspects of Caenorhabditis elegans development and behavior, encodes a clathrin-associated protein
}

\author{
Junho Lee, ${ }^{1}$ Gregg D. Jongeward, ${ }^{1}$ and Paul W. Sternberg ${ }^{2}$ \\ Howard Hughes Medical Institute and Division of Biology, California Institute of Technology, Pasadena, California \\ 91106 USA
}

\begin{abstract}
Our genetic analysis indicates that the unc-101 gene of Caenorhabditis elegans is required for many aspects of development and behavior, including negative regulation of vulval differentiation. We have cloned unc-101 and found that it encodes a homolog of the mammalian medium chains of clathrin-associated protein complexes located at the trans-Golgi and the plasma membrane, AP47 and AP50, respectively. Therefore, clathrin-mediated events might contribute to the negative regulation of vulval differentiation. Comparison of sequences, including a full-length sequence of a C. elegans AP50 homolog, reveals that UNC-101 is most closely related to AP47. Mouse AP47 and nematode UNC-101 proteins are functionally equivalent as assayed in transgenic nematodes. We have sequenced the mutant alleles of unc-101 identifed in various genetic screens and shown that all but one are deletions or nonsense mutations, suggesting that these alleles severely reduce unc-101 function.
\end{abstract}

[Key Words: C. elegans; unc-101; vulval induction; clathrin associated protein; AP47]

Received August 13, 1993; revised version accepted November 16, 1993.

Clathrin-coated pits and vesicles originate from the plasma membrane and the trans-Golgi in eukaryotic cells and mediate intracellular trafficking of membrane proteins (Fig. 1A; for review see Brodsky 1988; Keen 1990; Pearse and Robinson 1990; Schmid 1992). The main protein components in the coated vesicles are clathrin and their associated protein [adaptor, or assembly, protein (AP)] complexes. Whereas clathrin triskelions are common structural units to both the plasma membrane and the trans-Golgi coated vesicles, the AP complexes differ in these compartments, probably conferring specificity to clathrin vesicles. The trans-Golgiassociated protein complex AP-1 consists of two large chains, $\beta^{\prime}$ and $\gamma$; one medium chain, AP47; and one small chain, AP19 (Keen 1987; Ahle et al. 1988; Matsui and Kirchhausen 1990; Fig. 1B). The plasma membraneassociated protein complex AP-2 consists of two large chains, $\alpha$ and $\beta$; one medium chain, AP50; and one small chain, AP17. Although the large chains $\beta$ and $\beta^{\prime}$ are similar in sequence (Kirchhausen et al. 1989), the large chains $\alpha$ and $\gamma$ have a more diverged primary structure. Both medium and small chains of the plasma membraneassociated complex are homologous to their trans-Golgi counterparts. Coated vesicles of the plasma membrane

\footnotetext{
${ }^{1}$ These authors contributed equally to this paper.
}

${ }^{2}$ Corresponding author. are involved in the endocytosis of membrane proteins such as LDL receptor, transferrin receptor, and epidermal growth factor (EGF) receptor, whereas trans-Golgi coated vesicles are involved in the sorting of proteins, such as lysosomal enzymes, and are also thought to be involved in regulated secretion (e.g., Keen 1990; Pearse and Robinson 1990; Sorkin and Carpenter 1993).

In Saccharomyces cerevisiae, mutants deficient in a clathrin heavy chain are defective in retention of Kex2p protein in the Golgi apparatus (Seeger and Payne 1992). However, deletion of yeast small chains of the APs has little effect on cell growth, protein export, or endocytosis (H. Phan and G. Payne, pers. comm.). Similarly, deletion of a yeast medium chain does not result in any obvious phenotypic consequences, although there might be subtle phenotypes associated with these mutations (S.K. Lemmon, pers. comm.|. Because yeast is a single-cell organism and yeast clathrin vesicles and their APs may have more restricted functions than those in multicellular organisms, it is useful to examine the functions of coated vesicles in a multicellular organism. Bazinet et al. (1993) have recently found that clathrin heavy chain is essential in Drosophila.

unc-101 mutants of C. elegans were originally identified by their uncoordinated movement (D. Riddle, pers. comm.) and by abnormal uptake of dye by sensory neurons (E. Hedgecock, pers. comm.). We first identified 

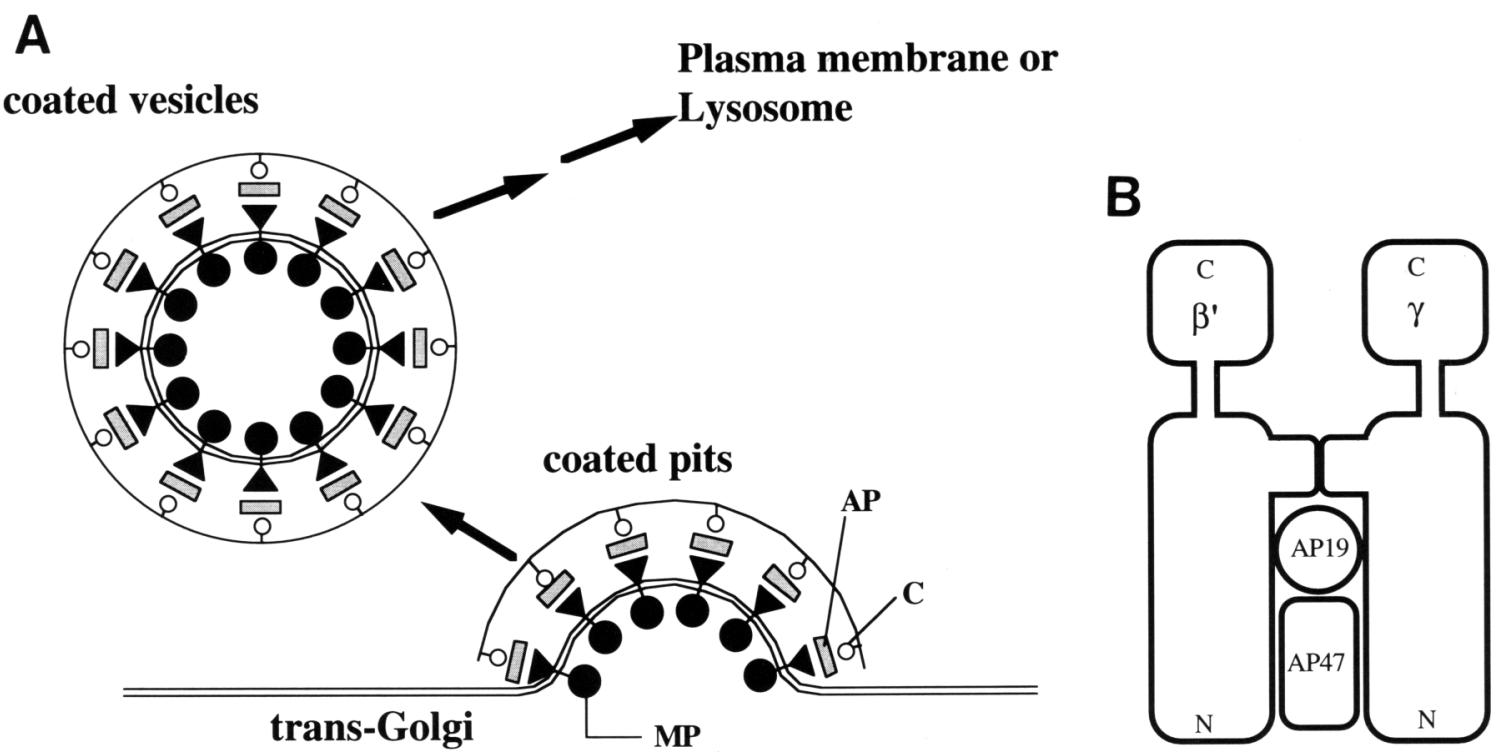

Figure 1. Clathrin vesicles and their associated protein complexes. $(A)$ Coated pits and vesicles. A coated pit is composed of membrane fraction, collected membrane proteins, and clathrin triskelion cage with its associated protein complex. Coated pits are invaginated to form coated vesicles, which travel to their destinations such as lysosome and the plasma membrane (modified from Pearse and Robinson 1990) (AP) AP complex; (C) clathrin; (MP) membrane protein. (B) Schematic structure of the clathrin-associated protein complex. AP-1 complex is composed of four different peptide chains: two large chains, $\beta, \gamma$; one medium chain, AP47; and one small chain, AP19. AP-2, not shown, has similar structure to that of AP-1 and is composed of two large chains, $\alpha, \beta$; one medium chain, AP50; and one small chain, AP17 (modified from Nakayama et al. 1991).

unc-101 alleles as extragenic suppressors of a reductionof-function mutation of let-23 (Aroian et al. 1990), an EGF receptor-like tyrosine kinase (G. Jongeward and P. Sternberg, in prep.). Here, we genetically analyze unc101 and provide evidence for its role in regulating vulval induction. We describe the molecular cloning of unc-101 and demonstrate its functional equivalence with the mouse AP47 protein. C. elegans thus provides an opportunity to use molecular genetics to study clathrin-associated protein complexes in a multicellular organism.

\section{Results}

\section{Pleiotropic effects of unc-101 mutations}

unc-101 mutations have pleiotropic effects on the behavior and development of C. elegans. Homozygous unc-101 animals have uncoordinated movement. They are very sluggish, do not respond to a light touch, and tend to coil. unc-101 animals also display abnormal uptake of the dye fluorescein-isothiocyanate (FITC) (E. Hedgecock, pers. comm.). In wild-type animals, six pairs of neurons in the amphid and two pairs of neurons in the phasmid can take up FITC (Hedgecock et al. 1985). In unc-101 animals, only one pair of amphid neurons stains, and the phasmid neurons stain faintly if at all. unc-101 mutant animals show irregularity in their defecation cycle (Thomas 1990). unc-101 animals also have male tail defects such as abnormal rays and spicules (S. Emmons; H. Chamberlin; both pers. comm.). In addition, unc-101 animals are subviable; about half of unc-101 animals do not survive to adulthood. These inviable animals typically arrest in late first larval (L1) stage but occasionally arrest in the $\mathrm{L} 2, \mathrm{~L} 3$, or L4 stages. We have isolated unc-101 mutations as suppressors of the vulvaless (Vul) phenotype of let23(sy1) hemizygotes (G. Jongeward and P. Sternberg, in prep.). Whereas unc-101 homozygotes have normal vulvae, they are defective in egg-laying, possibly resulting from their defect in late vulval morphogenesis.

\section{Genetic analysis of unc-101}

The unc-101 alleles $m 1$, rh6, sy108, and sy161 had been recovered previously from independent F2 screens, as described above. Although they were recovered in screens for different phenotypes, animals homozygous for these recessive alleles display an essentially identical spectrum of phenotypes. Because F2 screens could have failed to recover null alleles of unc-101 had the null phenotype been lethal, we performed two genetic tests to define a complete loss-of-function phenotype of unc-101. From these experiments we conclude that the existing unc-101 mutations reduce unc-101 activity severely.

We first identified a deletion of the unc-101 locus. We recovered sy216 by its failure to complement unc101(rh6) in a noncomplementation screen of 11,000 trimethylpsoralen (TMP)-mutagenized gametes (Materials and methods). Animals homozygous for sy216 arrest immediately after hatching without any apparent postembryonic cell divisions and live for several days before dying. This arrest phenotype is distinct from that of an- 
imals homozygous for all other unc-101 alleles, which arrest and die rapidly. Four observations suggest that sy216 is a deletion of the entire unc-101 locus. First, we were unable to amplify any unc-101 genomic DNA from sy216 homozygotes (see Materials and methods). Second, a rescuing cosmid (described below) could not rescue sy216 despite its ability to rescue the lethality of unc101(sy108). Third, Southern analysis failed to show any polymorphism in sy216/hIn1 heterozygotes using the rescuing cosmid as probe (data not shown), indicating that the deletion in sy216 is larger than $30 \mathrm{~kb}$. Finally, J.-Y. Ho and A. Rose (pers. comm.) have found that sy216 homozygotes are deleted for DNA on both sides of unc101.

We compared the existing alleles to the deletion of the unc-101 locus. The lethality of unc-101(sy216)/unc101(sy108) trans-heterozygotes is slightly enhanced (74\% inviable) with respect to sy108/sy108 homozygotes $(45 \%$ inviable). However, other phenotypes, such as suppression of the let-23(sy1) mutation, are not enhanced (see Materials and methods). These observations are consistent with sy108 being a severe reduction-offunction allele.

We then screened for new alleles of unc-101 under conditions that would allow the isolation of null alleles. About half of the progeny from homozygous unc-101 mothers die. However, all animals of the genotype unc101(sy108)/unc-101(sy216) from an unc-101(sy108)/ + mother are viable. Therefore, the maternal copy of unc$101(+)$ is sufficient to rescue the inviability of any new loss-of-function allele in trans to the visible allele sy108. From a screen of 20,000 Fl ethylmethane sulfonate (EMS)-mutagenized gametes, we recovered the sy241 allele. sy241 homozygotes are viable and have phenotypes essentially identical to other unc-101 homozygotes.

unc-101 is a negative regulator of the vulval induction pathway

In C. elegans, a signal from the gonadal anchor cell induces three of six vulval precursor cells (VPCs) to generate vulval tissue (for review, see Horvitz and Sternberg 1991). Reduction-of-function mutations in the lin-3, let23, sem-5, let-60, or lin-45 genes result in a Vul phenotype. Each of these genes encodes a C. elegans homolog of a mammalian signaling or signal transduction protein: lin-3 encodes an EGF-like growth factor; let-23, an EGFreceptor homolog; sem-5, an SH3-SH2-SH3 adaptor; let60, a Ras protein; and lin-45, a Raf serine/threonine kinase (for review, see Sternberg 1993). Other genes (lin-2, lin-7, and lin-10) are also required for vulval induction. Genetic pathway analysis suggests that lin-3 acts via let23 , which acts via sem-5. sem-5 acts via let -60 , which acts via lin-45 to stimulate vulval differentiation (for review, see Sternberg 1993). lin-2, lin-7, and lin-10 are not absolutely required for this signaling; apparent null mutations (Ferguson and Horvitz 1985) act like partial reduction-of-function mutations of let-23. These three genes appear to act at the let-23 step in the signaling pathway.
We recovered two alleles of unc-101 (sy108 and sy161) as suppressors of the Vul phenotype of the weak let-23 allele, sy1. Although mutations at the unc-101 locus confer no defect in the extent of vulval differentiation in the absence of another mutation, averaging three VPCs forming vulval tissue per animal, several non-null mutations of let-23 are suppressed by an unc-101 mutation (Table 1). let-23(sy1), the allele used in the original screen, is suppressed strongly. let-23(sy1) animals average one VPC per animal forming vulval tissue, whereas unc-101;let-23(sy1) animals are hyper-responsive to the signal, averaging 3.6 VPCs forming vulval tissue per animal. After ablation of the gonad of the unc101(sy108);let-23(sy1) double mutants, none of seven animals displayed vulval differentiation, indicating that vulval differentiation in the double mutants still requires the signal from the gonad as in unc-101 mutant $(n=6)$ or wild-type animals. A more severe mutation, let-23(sy12), is suppressed from an average of less than one to nearly three VPCs per animal forming vulval tissue. Another severe allele, let-23(sy97), is only slightly suppressed, if at all. The let-23(n1045) allele is enhanced by an unc-101 mutation (Table 1; see Discussion).

To determine whether the interaction of unc-101 is limited to let-23, we constructed a series of double mutants and examined their extent of vulval differentiation (Table 1). unc-101 mutations suppress reduction-of-function mutations in several of the genes required for vulval differentiation. Strong reduction-of-function alleles of lin-2, lin-7, or lin-10 cause a Vul phenotype with an average of $0.4-0.9$ VPC per animal undergoing vulval differentiation. unc-101 mutations can suppress the Vul phenotype of each of these mutants to an average of 3.4 VPCs differentiating per animal. In contrast to this strong suppression, reduction-of-function mutations of lin-3 (the inductive signal) or lin-45 (a downstream signal transducer) are only partially suppressed by an unc-101 mutation. Specifically, lin-3(n378) homozygotes average less than one VPC per animal forming vulval tissue, whereas unc-101;lin-3(n378) double mutants average slightly more than two VPCs forming vulval tissue per animal. Animals bearing lin-3(e1417) also display this partial suppression. lin-45(sy96) animals average 1 VPC forming vulval tissue, whereas unc-101;lin-45(sy96) animals average 1.9 VPC forming vulval tissue. A dominant interfering mutation of let-60 ras is not suppressed. We conclude that unc-101 is a negative regulator of vulval differentiation. A simple interpretation of the spectrum of unc-101 suppression is that unc-101 normally acts closer to the let-23 step than at either upstream (lin-3) or downstream (let-60, lin-45) steps in this signaling pathway.

let-23 is necessary for several aspects of development (Aroian and Sternberg 1991; Aroian et al. 1993a). unc-101 mutations suppress let-23 mutant phenotypes in a subset of these tissues. Complete loss-of-function alleles of let-23 confer L1 larval lethality on all homozygotes. An unc-101 mutation does not suppress the lethality of homozygotes for complete loss-of-function alleles of let-23 nor the incompletely penetrant lethality conferred by 
Table 1. Interaction of unc-101 with other genes in the vulval induction pathway

\begin{tabular}{|c|c|c|c|c|}
\hline \multirow[b]{2}{*}{ Genotype } & \multicolumn{2}{|c|}{ unc-101(+) } & \multicolumn{2}{|c|}{ unc-101(sy108) } \\
\hline & $\begin{array}{l}\text { VPCs/animal } \\
\text { differentiating }\end{array}$ & $\begin{array}{l}\text { animals with } \\
>3 \text { VPCs } \\
\text { differentiating (\%) }\end{array}$ & $\begin{array}{l}\text { VPCs/animal } \\
\text { differentiating }\end{array}$ & $\begin{array}{l}\text { animals with } \\
>3 \text { VPCs } \\
\text { differentiating }(\%)\end{array}$ \\
\hline+ & 3.0 & 0 & 3.0 & 0 \\
\hline $\operatorname{lin}-3(e 1417)$ & 0.8 & 0 & 1.4 & 0 \\
\hline $\operatorname{lin}-3(n 378)$ & 0.8 & 0 & 2.1 & 0 \\
\hline let-23(sy97) & 0.0 & 0 & 0.06 & 0 \\
\hline let-23(sy12) & 0.02 & 0 & 2.9 & 10 \\
\hline let-23(sy1) & 0.8 & 0 & 3.6 & 40 \\
\hline let-23(n1045) $\left(15^{\circ} \mathrm{C}\right)$ & 1.1 & 0 & 0.2 & 0 \\
\hline let-23(n1045) $\left(20^{\circ} \mathrm{C}\right)$ & 2.5 & 10 & 0.6 & 0 \\
\hline let-23(n1045) $\left(25^{\circ} \mathrm{C}\right)$ & 3.4 & 40 & 2.0 & 0 \\
\hline $\operatorname{lin}-2(n 768)$ & 2.8 & 0 & 3.2 & 20 \\
\hline $\operatorname{lin}-2(e 1309)$ & 0.5 & 0 & 3.4 & 40 \\
\hline $\operatorname{lin}-7(e 1413)$ & 0.9 & 0 & 3.4 & 40 \\
\hline $\operatorname{lin}-10(e 1439)$ & 0.5 & 0 & 3.4 & 30 \\
\hline let-60(sy100 dn) & 0.0 & 0 & 0.0 & 0 \\
\hline $\operatorname{lin}-45($ sy96) & 0.9 & 0 & 1.9 & 10 \\
\hline
\end{tabular}

Vulval differentiation in $\sim 20$ animals per each genotype was examined using Nomarski optics. A wild-type animal has 3 VPCs induced. Fewer than 3 VPCs induced result in a Vul phenotype. When $>3$ VPCs generate vulval tissue, a normal vulva and pseudovulval tissue are usually produced.

the sy97 mutation. The sterility of the allele let-23(sy12) is partially suppressed. Whereas all viable let-23(sy12) hermaphrodites are sterile, $\sim 65 \%$ of unc-101;let23(sy12) animals are at least slightly fertile. The fact that unc-101 mutations do not suppress a complete loss-offunction mutation for this pathway suggests that unc101 mutations do not simply allow a bypass of this signaling pathway but, rather, relieve some negative regulation that impinges on the signaling pathway.

\section{Molecular cloning of the unc-101 locus}

To clone unc-101, we correlated the genetic and physical maps around this locus by identifying and mapping transposon polymorphisms (Fig. 2). Multipoint restriction fragment length polymorphism (RFLP) mapping (Ruvkun et al. 1989) was used to map two Tc1 transposon polymorphisms, syP6 and syP7, with respect to the genetic markers unc-75, ced-1, unc-101 and unc-59. No recombination events were observed between $s y P 7$ and unc-101 in the 77 recombinants, indicating that $s y P 7$ is very close to unc-101. Because the physical distance between the two polymorphisms is $\sim 600 \mathrm{~kb}$ and because syP7 is inseparable from the unc-101(+) marker, we tested genomic cosmid clones within $100 \mathrm{~kb}$ of $s y P 7$ for their ability to complement the unc-101 phenotype.

We identified a genomic cosmid clone that can rescue the uncoordinated phenotype of unc-101 mutations when introduced as an extrachromosomal multicopy transgene by microinjection (Fig. 2). Only cosmid W05A3 was able to rescue unc-101(sy108). A 6.3-kb subclone of W05A3, pIL5, is the smallest genomic fragment capable of rescuing the uncoordinated phenotype. The pJL5 subclone rescues at least three other phenotypes of unc-101(sy108): lethality, defective uptake of FITC, and suppression of let-23(sy1) Vul phenotype. The partial lethality of unc-101(sy108) was rescued in the transgenic animals carrying pJL5 from $47 \%$ to $72 \%$ survival (Table 2). pIL5 also suppresses the FITC staining defect of unc101(sy108) animals (data not shown). The vulval differentiation of unc-101(sy108);1et-23(sy1) animals carrying pJL5 as a transgene was lowered from 3.6 VPCs induced per animal, as seen in unc-101(sy108);1et-23(sy1) double mutants lacking the transgene, to $0.8 \mathrm{VPC}$ induced per animal $(n=20)$, an extent similar to that of let-23(sy1) single mutants. Therefore, pJL5 also rescues the suppression of the let-23(sy1) mutation caused by unc101(sy108). We did not test other phenotypes associated with unc-101 mutations for rescue.

\section{Genomic and cDNA structure of unc-101}

We isolated a full-length cDNA clone from a cDNA library (Barstead and Waterston 1989) using the pJL2 plasmid as probe (Fig. 2) and determined its nucleotide sequence (Fig. 3A). There are three in-frame stop codons $5^{\prime}$ to the putative ATG codon. Also, the $5^{\prime}$ end of the cDNA sequence has 7 nucleotides that are identical to the $3^{\prime}$ end sequence of the trans-spliced leader sequence SL1 (Krause and Hirsh 1987), suggesting that this cDNA has a full-length coding sequence and that unc-101 is a transspliced gene. A polyadenylation signal, AATAAA, is present at nucleotide 1844 . The nucleotide sequence predicts that the unc-101 gene product is a protein of 422 amino acids. 
Figure 2. Cloning of the unc-101 locus. (A) Genetic and physical map near unc101. The genetic distance between ced-1 and unc-101 is $\sim 5$ map units. $(\nabla)$ RFLPs. syP6 is in yeast artificial chromosomes (YACs) $\mathrm{Y} 38 \mathrm{H} 7$ and $\mathrm{Y} 53 \mathrm{C} 10$, the same YAC that contains ced-1. syP7 is in YACs Y45E10, Y45A5, and Y61E1, and cosmids K02B8 and C54C8, a position $\sim 600 \mathrm{~kb}$ from syP6 and genetically inseparable from the unc-101( +) marker. The W05A3 cosmid, but not the other four cosmids, had the ability to rescue Unc phenotype of unc-101(sy108). (B) Map of W05A3 cosmid and its subclones. pJL5, a 6.3-kb subclone, is the smallest genomic region capable of rescuing. pJL3 does not rescue the phenotype, suggesting that this restriction enzyme site disrupts the unc-101 gene. Restriction sites shown are as follows: (S) SpeI; (N) NdeI; (H) HindIII; (P) PstI; (A)ApaI.

We inferred the genomic structure of unc-101 by partial genomic sequence data and PCR with primers specific to cDNA regions (Fig. 3B). The unc-101 cDNA is divided into seven exons. The smallest rescuing plasmid, pIL5, has 95 nucleotides 5 ' to the SLl acceptor sequence.

\section{Sequences of unc-101 mutant alleles}

To confirm our identification of the unc-101-coding region, we analyzed the lesions associated with unc-101 alleles. We determined the locations of the mutations of

Table 2. Complementation of unc-101 mutation by $p / L 5$ : suppression of lethality

\begin{tabular}{lcccc}
\hline $\begin{array}{l}\text { Genotype } \\
\text { of parental } \\
\text { chromosome }\end{array}$ & $\begin{array}{l}\text { Transgene } \\
\text { present }\end{array}$ & $\begin{array}{l}\text { Viability } \\
(\%)\end{array}$ & \multicolumn{2}{c}{$\begin{array}{l}\text { Stability of } \\
\text { transgene } \\
(\%)\end{array}$} \\
\hline$+1+$ & + & 91 & 390 & 41 \\
sy108/+ & + & 98 & 261 & 31 \\
sy108/sy108 & + & 72 & 241 & 32 \\
sy108/sy108 & - & 47 & 91 & N.A. \\
\hline
\end{tabular}

Eggs were transferred to new plates from hermaphrodites of three different genotypes, and the number of surviving adults and rolling animals were counted after 3 days. (Viability) Number of adults $/ N ;(N)$ number of eggs picked; (stability) number of rollers $/ N$. The pIL5 plasmid, when maintained in the transgenic animals with a dominant version of rol-6 as a marker, can enable the animals to survive (see text for details). sy108 homozygotes were Unc animals from the transgenic line that lost the transgene. eight alleles of unc-101 (Fig. 3B). We determined the mutations by directly sequencing PCR-amplified DNA preparations from genomic DNA or single mutant animals (Kretz et al. 1989). All but one allele are predicted to produce truncated proteins due to either a deletion or a nonsense mutation. sy108 is a deletion of 115 nucleotides in exon 3 and intron 3 and an insertion of $8 \mathrm{nu}-$ cleotides at the deletion point. sy237, sy241, $m 1$, and $r h 6$ are nonsense mutations, encoding truncated proteins. sy237 is a $\mathrm{G} \rightarrow \mathrm{A}$ transition at nucleotide 550 , changing a TGG codon to a TAG stop codon. sy241 is a $\mathrm{C} \rightarrow \mathrm{T}$ transition mutation at nucleotide 1284 , changing a CAA codon to a TAA stop codon. $m 1$ is a $\mathrm{C} \rightarrow \mathrm{T}$ mutation at nucleotide 1314 , changing a CAA codon to a TAA stop codon. rh6 is another $\mathrm{C} \rightarrow \mathrm{T}$ mutation at nucleotide 1086 , changing CAA to TAA. sy161, the only missense mutation, is a $\mathrm{C} \rightarrow \mathrm{T}$ mutation at nucleotide 552 , changing CGC (arginine) to TGC (cysteine). This arginine residue is conserved in both AP47 and AP50 (see below; Fig. 4A). We were unable to amplify by PCR any genomic DNA from sy216 homozygotes, suggesting that this $\mathrm{mu}$ tation is a deletion of the entire gene (see Materials and methods|.

\section{unc-101 encodes a clathrin-associated protein}

The predicted translation product of the unc-101 cDNA sequence is highly similar to two mammalian proteins, mouse AP47, the medium chain of the trans-Golgi-associated, clathrin-associated complex AP-1, and rat AP50, the medium chain of plasma membrane-associated, 
GAAGTTTGAGTATTTTCCAGTAGCTGCCACGTGGAATTTGACGATTTTAACGAGAAAATC 60 GCAAAAAATCGTCGAAAATGGCGACTTCCGCCATGTTTATACTGGATTTGAAGGGAAAAA 120 $\begin{array}{lllllllllllllllllll}M & A & T & S & A & M & F & I & L & D & L & K & G & K\end{array}$ CGATAATTTCTCGAAATTATCGCGGAGACATCGACATGACGGCAATCGATAAATTCATTC 180 $\begin{array}{llllllllllllllllllll}T & I & I & S & R & N & Y & R & G & D & I & D & M & T & A & I & D & K & F & I\end{array}$ ATTTACTCATGGAAAAAGAGGAAGAAGGCTCGGCAGCGCCCGTTTTGACCTATCAGGACA 240 $\begin{array}{llllllllllllllllllllll}H & L & L & M & E & K & E & E & E & G & S & A & A & P & V & L & T & Y & Q & D\end{array}$ CGAATTTCGTGTTTATCAAGCACACAAATATTTATTTGGTCTCAGCATGCCGTTCAAACG 300 $\begin{array}{lllllllllllllllllllllllllllll}T & N & F & V & F & I & K & H & T & N & I & Y & L & V & S & A & C & R & S & N\end{array}$ TCAACGTCACAATGATTTTGTCATTTTTGTACAAATGCGTCGAAGTTTTCTCCGAATATT 360 $\begin{array}{lllllllllllllllllllllllllll}V & N & V & T & M & I & L & S & F & L & Y & K & C & V & E & V & F & S & E & Y\end{array}$ TCAAAGATGTCGAAGAGGAGTCGGTICGCGACAATTTTGTCGTTATCTATGAACTTTTGG 420 $\begin{array}{lllllllllllllllllllllllll}F & K & D & V & E & E & E & S & V & R & D & N & F & V & V & I & Y & E & L & L\end{array}$ ACGAAATGATGGATTTCGGGTTCCCACAGACGACTGAGAGTCGAATTCTACAAGAATACA 480 $\begin{array}{llllllllllllllllllllll}D & E & M & M & D & F & G & F & P & Q & T & T & E & S & R & I & L & Q & E & Y\end{array}$ TCACACAAGAAGGTCAAAAACTAATTTCGGCACCCCGTCCCCCGATGGCAGTGACAAATG 540 $\begin{array}{lllllllllllllllllllllllll}I & T & Q & E & G & Q & K & L & I & S & A & P & R & P & P & M & A & V & T & N\end{array}$ CCGTCTCATGGCGCTCTGAAGGCATAAAATACCGAAAAAACGAGGTTTTCCTGGACGTAA 600 $\begin{array}{lllllllllllllllllllll}A & V & S & W & R & S & E & G & I & K & Y & R & K & N & E & V & F & L & D & V\end{array}$ TCGAAAGTGTGAACATGTTGGCCAGCGCCAACGGTACCGTACTTCAATCGGAAATTGTTG 660 $\begin{array}{llllllllllllllllllll}I & E & S & V & N & M & L & A & S & A & N & G & T & V & L & Q & S & E & I & V\end{array}$ GAAGCGTTAAAATGCGTGTCTATCTTACCGGAATGCCTGAACTTCGGCTGGGTCTTAACG 720 $\begin{array}{llllllllllllllllllllll}G & S & V & K & M & R & V & Y & L & T & G & M & P & E & L & R & L & G & L & N\end{array}$ ATAAAGTACTTTTTGAGGGCAGTGGACGCGGAAAAAGCAAATCTGTGGAACTGGAAGACG 780 $\begin{array}{lllllllllllllllllllll}D & K & V & L & F & E & G & S & G & R & G & K & S & K & S & V & E & L & E & D\end{array}$ TGAAATTTCATCAATGTGTACGCCTGTCGCGTTTTGACACGGATCGAACGATCTCCTTCA 840 $\begin{array}{lllllllllllllllllllllll}V & K & F & H & Q & C & V & R & L & S & R & F & D & T & D & R & T & I & S & F\end{array}$ TACCGCCCGACGGAGCATTTGAGCTTATGAGCTATCGATTAACAACCGTGGTGAAGCCGC 900 $\begin{array}{llllllllllllllllllllll}I & P & P & D & G & A & F & E & L & M & S & Y & R & L & T & T & V & V & K & P\end{array}$ TGATCTGGATCGAGACAAGCATCGAACGTCACAGTCACAGCCGTGTCTCGTTTATAATCA 960 $\begin{array}{llllllllllllllllllll}L & I & W & I & E & T & S & I & E & R & H & S & H & S & R & V & S & F & I & I\end{array}$ AAGCGAAATCACAATTCAAACGGCGCTCCACTGCTAATAACGTGGAAATCATTATTCCAG 1020 $\begin{array}{lllllllllllllllllllll}K & A & K & S & Q & F & K & R & R & S & T & A & N & N & V & E & I & I & I & P\end{array}$ TCCCGTCGGACGCTGATTCACCGAAATTCAAGACAAGCATCGGTTCGGTGAAGTATACGC 1080 $\begin{array}{lllllllllllllllllllllllll}V & P & S & D & A & D & S & P & K & F & K & T & S & I & G & S & V & K & Y & T\end{array}$ CCGAGCAATCGGCCTTCGTATGGACTATTAAGAATTTTCCCGGCGGAAAAGAGTACCTTT 1140 $\begin{array}{lllllllllllllllllllll}P & E & Q & S & A & F & V & W & T & I & K & N & F & P & G & G & K & E & Y & L\end{array}$ TGACCGCCCATCTATCTCTACCGTCTGTGATGAGTGAAGAGTCTGAAGGACGGCCGCCGA 1200 $\begin{array}{llllllllllllllllllllllll}\text { L } & T & A & H & \text { L } & S & L & P & S & V & M & S & E & E & S & E & G & R & P & P\end{array}$ TTAAAGTCAAATTTGAAATTCCGTATTTTACGACCAGCGGCATTCAGGTCCGTTATCTGA 1260 $\begin{array}{llllllllllllllllllll}I & K & V & K & F & E & I & P & Y & F & T & T & S & G & I & Q & V & R & Y & L\end{array}$ AAATCATCGAGAAAAGAGGATATCAAGCATTGCCGTGGGTCCGCTACATTACTCAAAATG 1320 $K \begin{array}{llllllllllllllllllllllll}K & I & E & K & R & G & Y & Q & A & L & P & W & V & R & Y & I & T & Q & N\end{array}$ GAGAATACGAGATGCGGATGAAATAATTCCTGAAAAAATTACCTAAATTCATATTTTATT 1380 $G \quad E \quad Y \quad E \quad M \quad R \quad M \quad K$ *

GTATTTTATTCCCAATTTTACTCTTAATTTTTGGAATTTTTTATGAAAAATTGGTGAAAA 1440 ACGACCGTGGCCGAGTTTTTGGAAAATTTGGAGGCTAGGCCACCATGCTTCCAGTGGTGG 1500 GCTAACT'TTTCGAAAATCCTAGCCACGGCCCCGTTTTCCATCAATTTTTGCCTCTTTTAA 1560 TGTCAAACATCTCCAATTTTTTCTGTGAAAATTTAATGCTCCGCGAGCTGCTCCCCGGCT 1620 CAAAACATGTGTTGTGTGTGCTCTTTCCCCTGATGACCCCGAACTCTATTTTTTTTTGTT 1680 CGAAAATTTTTATTTTATTTTTCCCACCGATTTATTTATTGATTTTTATCGCATAATTTA 1740 GATTTTTTCCAGAAAAACGAGTTTTTTCCCTTTCCCACCAGCATAATTTTCTCACCACAT 1800 GGATCCTCATCAATTTTTCCCGTTTTCCTTTTTTCAGTAAATCAATAAAATTTTCTGTCA 1860 TAATTAAAAAAAAAAAAAAAAAC 1883

B

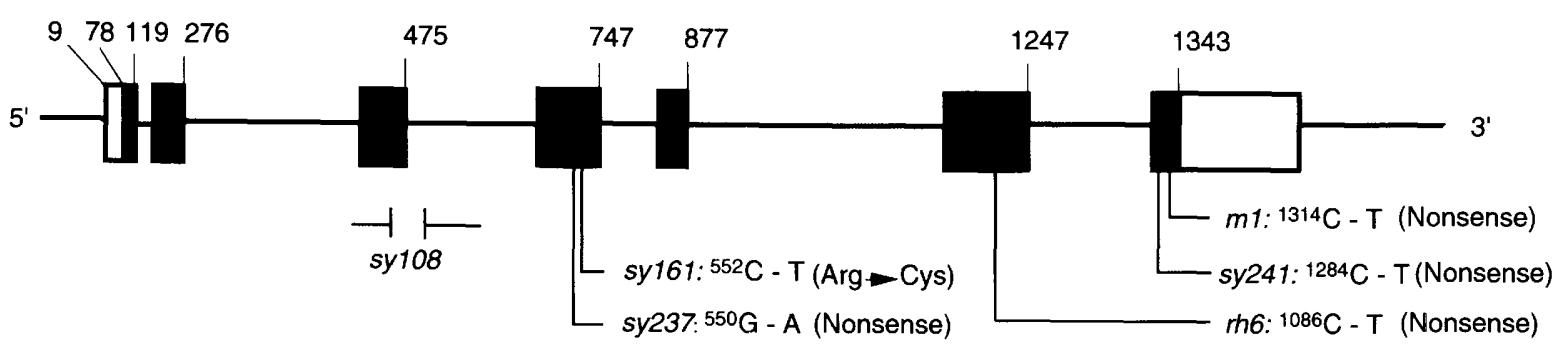

Figure 3. cDNA sequence and genomic structure of unc-101. (A) unc-101 cDNA sequence and its predicted translation product. The region identical to the $3^{\prime}$ end of SL1 sequence is underlined. The start and stop codons, and a polyadenylation signal sequence are also underlined. The sites for the construction of the mammalian AP47 hybrid gene are underlined with arrows. The first nucleotide, G, is the last nucleotide of the vector. $(B)$ Genomic structure of unc-101. unc-101 has seven exons and six introns. The size of introns varies from $50 \mathrm{bp}$ to $1.0 \mathrm{~kb}$. The numbers above the structures represent the first nucleotide of the cDNA after SLI sequence, the translation starting point, and the last nucleotides of exons. The locations and characteristics of mutations are marked as such. 


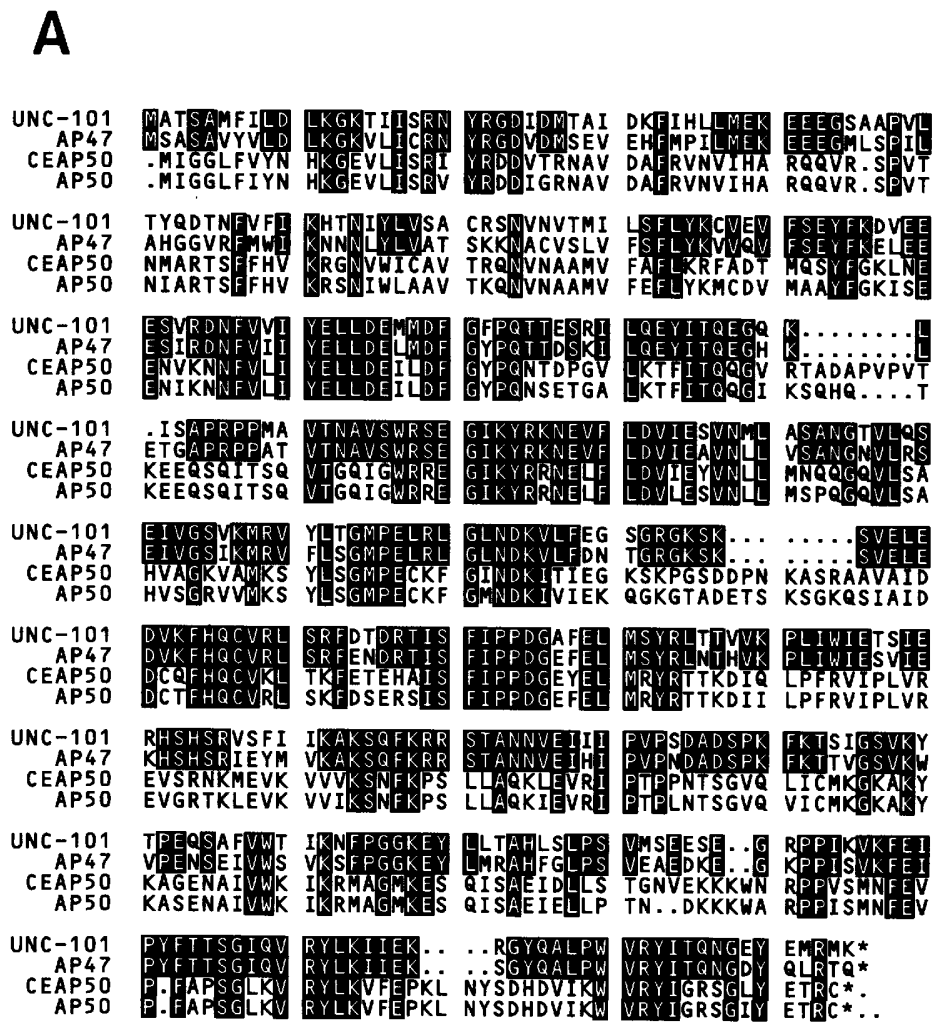

B

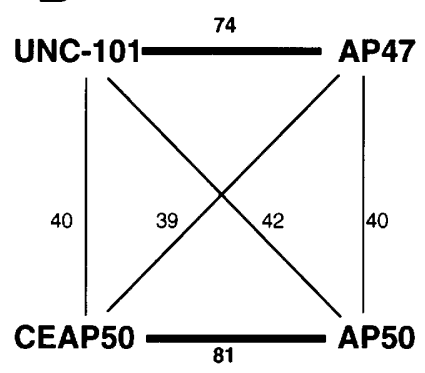

Figure 4. UNC-101 is a clathrin-associated protein. $(A)$ Amino acid sequence alignment of homologs of UNC101. AP47 and AP50 are the medium chains of trans-Golgi clathrin-associated protein complex and plasma membrane clathrin-associated protein complex, respectively (Thurieau et al. 1988; Nakayama et al. 1991). CEAP50 is an AP50 homolog in C. elegans (this study). This alignment only highlights the residues that are identical between UNC-101 and AP47 or between all four homologs. The GenBank accession numbers are L26290 for CEAP50 and L26291 for UNC-101. (B) Diagram of the comparison of identity among the homologs of medium chains of clathrin-associated complexes. The numbers represent the identity at amino acid sequence level. The most prominent homologies are marked by boldface lines. UNC-101 and AP47 are more similar to each other than to AP50 and CEAP50. clathrin-associated complex AP-2 (Fig. 4; Thurieau et al. 1988; Nakayama et al. 1991) UNC-101 is also similar to a yeast protein, Yap54, the yeast homolog of AP47 (Nakayama et al. 1991). UNC-101 protein is $74 \%$ identical to AP47 and $42 \%$ identical to AP50, suggesting that UNC-101 is a homolog of the mammalian AP47.

To test whether UNC-101 is a homolog of AP47, we sought to identify AP50 homologs in C. elegans. The C. elegans genome sequencing consortium has identified a cDNA encoding a homolog of AP50 (Waterston et al. 1992). Using this cDNA clone as probe, we isolated three more cDNA clones and determined their sequences. All three were products of a single gene. One of the three cDNA clones had a presumably full-length coding sequence, based on homology with other medium chains. The comparison of the amino acid sequence of this predicted protein with those of other homologs revealed that it is $81 \%$ identical to AP50 and $40-42 \%$ identical to AP47 homologs, indicating that this sequence is an AP50 homolog (Fig. 4). On the basis of the extent of the amino acid sequence identity, we believe that unc-101 encodes a C. elegans homolog of AP47 protein.

We physically mapped the AP50 homolog to the X chromosome by yeast artificial chromosome (YAC) grid hybridization and identified a cosmid (T16D11) that contains the coding region of AP50 homolog in the middle of its insert (data not shown). When injected into unc101(sy108) animals, this cosmid did not rescue the Unc phenotype of unc-101(sy108) animals. This negative result is consistent with unc-101 being a homolog of AP47.
The function of AP47 is conserved in mammals and nematodes

Because the amino acid sequence of unc-101 is similar to that of the mammalian homolog AP47, it is conceivable that their function has been conserved during evolution. To test this hypothesis, we examined whether the mammalian AP47 gene can rescue the phenotype of unc-101 animals. We constructed a hybrid gene with unc-101 genomic DNA and mouse AP47 cDNA (Fig. 5). In the mouse/nematode hybrid construct, 298-amino-acid residues of 422 amino acids are from the mouse cDNA. We also constructed an unc-101 cDNA hybrid gene that is identical to the AP47 hybrid except that it has unc-101 cDNA instead of AP47 cDNA. As a control, we constructed an unc-101 gene deleted for the 298 amino acids replaced by the cDNAs. Both hybrid genes rescue the Unc phenotype of unc-101(sy108) animals (Fig. 6); the control construct does not. The extent of vulval differentiation of the transgenic unc-101(sy108);let-23(sy1) animals carrying the AP47 hybrid gene was an average of 2.0 VPCs per animal $(n=10)$, compared with 3.6 VPCs of unc-101(sy108);let-23(sy1) animals without the transgene $(n=20)$, indicating that the suppression of the let23(sy1) Vul phenotype was also rescued (Fig. 7). Thus, mouse AP47 and C. elegans UNC-101 are not only similar in sequence but also functionally interchangeable. Rescue of the lethality associated with unc-101(sy108) animals was not checked because of low transmission of the transgene. 
$1 \mathrm{~kb}$

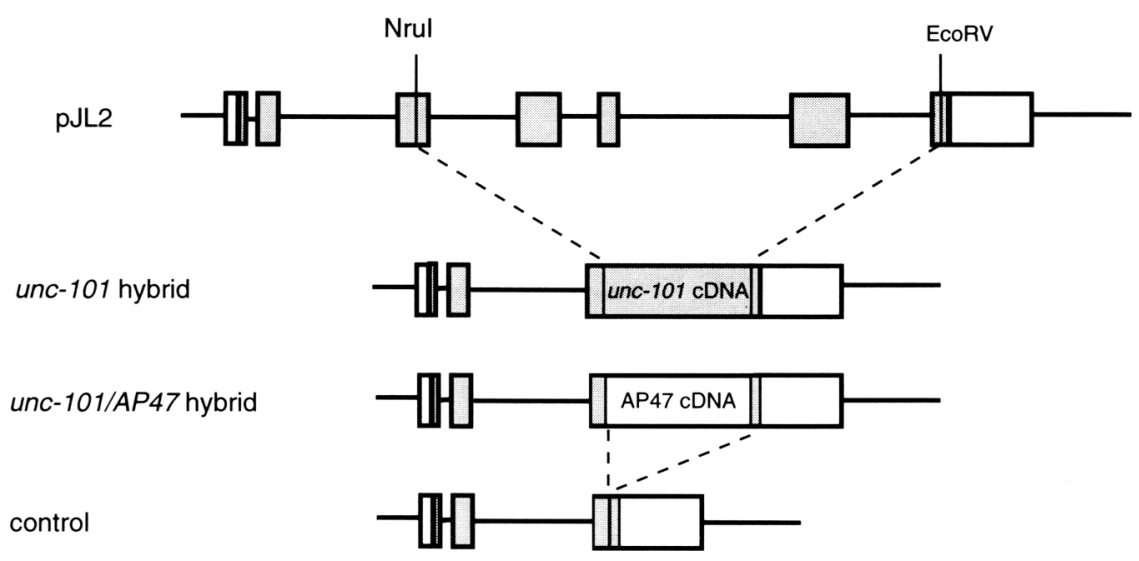

Figure 5. Schematic of mouse/nematode hybrid gene construction. The NruI-EcoRV fragment of the rescuing plasmid pJL2 was replaced with the corresponding cDNA fragments from unc-101 cDNA clone or mouse AP47 cDNA clone in the unc-101 hybrid gene and in the unc-101/AP47 hybrid gene, respectively.

\section{Discussion}

We have analyzed the C. elegans unc-101 gene genetically and molecularly. unc-101 mutations not only cause a variety of behavioral and developmental defects but also suppress the Vul phenotype of weak alleles of let-23, a C. elegans EGF receptor homolog required for vulval induction. We have cloned the unc-101 gene and have shown that it encodes a homolog of AP47, the medium chain of the trans-Golgi clathrin-associated complex. We also determined the cDNA sequence of a homolog of AP50, the medium chain of plasma membrane-associated complex. Sequence comparison clearly indicates that UNC-101 is an AP47 homolog. This homology was confirmed by the fact that a nematode unc-101/mouse AP47 hybrid gene could functionally replace the nematode unc-101 gene. unc-101 provides the only known case in which mutations of clathrin AP genes cause visible phenotypes. Because unc-101 mutations have pleiotropic phenotypes and because mammalian AP47 and nematode UNC-101 are functionally interchangeable, one can study the function of clathrin-coated vesicles in the regulation of signal transduction. Further study of unc-101 and homologs of other components of the clathrin-coated vesicles on the plasma membrane and the trans-Golgi compartment will help us to understand the nature of the coated vesicles and their roles in a wellcharacterized signal transduction pathway.

\section{Null phenotype of unc-101}

We analyzed alleles of unc-101 recovered in several screens to define the loss-of-function phenotype of this locus. We are convinced that the visible alleles, exemplified by sy108 severely reduce and possibly eliminate unc-101 function for the following reasons: (1) sy108 in trans to a deletion of the locus sy216 has a similar phenotype to sy108 homozygotes; (2) in a screen that should be able to recover null alleles of unc-101, we obtained an allele with identical phenotypes to the existing alleles rather than homozygous lethal allele, for example; and
(3) all but one allele are deletions or nonsense mutations, encoding truncated and probably nonfunctional proteins.

The subviability of unc-101 mutant animals could be explained by residual function of unc-101 by the aminoterminal fragments. However, multicopy transgenes carrying just the amino terminus of the protein do not rescue unc-101 mutants, although we cannot exclude readthrough of stop codons. Another possibility is general sickness caused by the cumulative effects of many unc-101 defects. It is also possible that there is a partially redundant homolog of AP47. Our preliminary observations suggest that there is another homolog of AP47 in $C$. elegans (J. Lee and P. Sternberg, unpubl.), but whether this protein is expressed in the same cells as UNC-101 is yet to be determined.

\section{Structure and function of AP50/AP47 proteins}

It is not surprising that AP50 and AP47 sequences are similar (40\% identical over 422 amino acids; Nakayama et al. 1991), because other components of the clathrinassociated complexes also are similar. Mammalian small chains AP17 and AP19 are $44 \%$ identical, and large chains $\beta$ and $\beta^{\prime}$, show the highest degree of identity of $84 \%$ (Kirchhausen et al. 1991). The exception is $\alpha$ and $\gamma$ large chains, which are only $29 \%$ identical. Given the extent of similarity between the components of the APs, it is conceivable that $\beta$ and $\beta^{\prime}$ chains have common functions, such as binding to clathrin trimers, and that the medium and small chains have some unique and some common functions. In contrast, $\alpha$ and $\gamma$ large chains may have unique functions such as binding to particular membrane proteins.

Amino acid residues conserved in both AP47 and AP50 might have common functions such as interaction with clathrin trimers and membrane components. These residues are distributed throughout the peptide sequence. Amino acid residues specifically conserved in either AP47 or AP50 homologs may be important for their unique functions, such as interaction with particular 


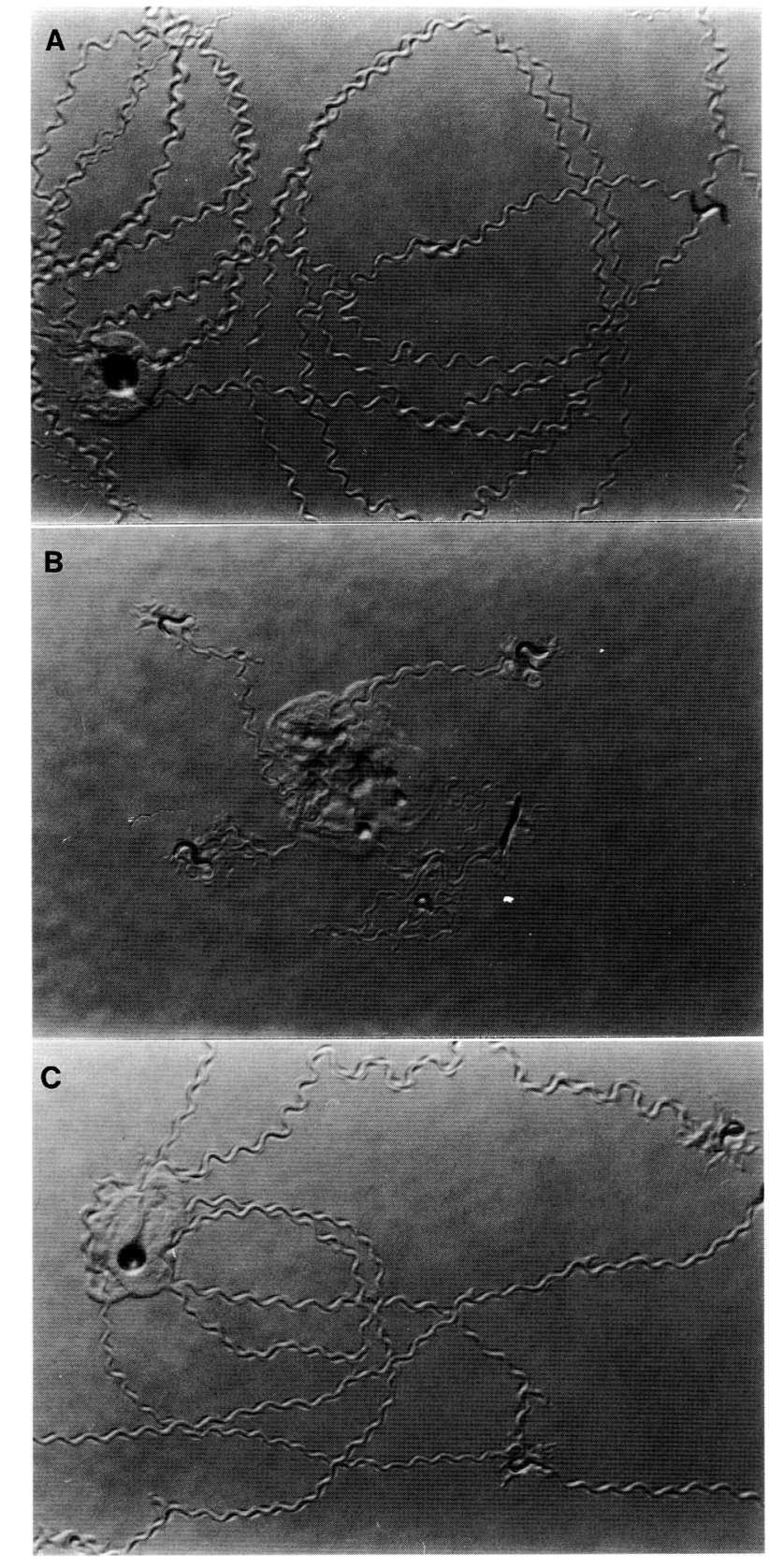

Figure 6. Rescue of Unc phenotype by the mouse/nematode hybrid gene. The tracks that the worms create by moving on the bacterial lawn are shown. (A) N2 wild type, 5 min after transfer; (B) unc-101(sy108), 40 min after transfer; $(C)$ transgenic unc101(sy108) carrying the AP47 hybrid as a transgene, 5 min after transfer. The uncoordinated movement of unc-101(sy108) is suppressed by the transgene.

membrane proteins, or other components of their own type of AP complex. These residues are also distributed throughout the peptide sequence, making it difficult to predict the domain structure of the proteins. So far, we have not found residues important for AP47-specific function. sy161 is the only missense mutation, and it occurs at an amino acid conserved in both the AP47 and AP50 proteins. The $m 1$ and $r h 6$ alleles encode proteins missing just a few carboxy-terminal residues but confer identical phenotypes to other alleles, suggesting that the carboxy-terminal residues are important for function, stability, or regulation.

\section{Negative regulation of $\mathrm{C}$. elegans vulval induction}

We have provided evidence that unc-101 also acts as a negative regulator of vulval differentiation. Mutations of unc-101 strongly suppress defects associated with mutations in genes such as let-23, lin-2, lin-7, and lin-10 that are required for vulval differentiation, suggesting that one wild-type function of unc-101 is to negatively regulate the process of vulval induction. The loss of unc-101 activity in an otherwise wild-type animal confers no defect in the extent of vulval differentiation, suggesting that unc-101 acts to modulate the inductive signaling pathway, rather than acting as a target of the signal transduction pathway. Consistent with this, unc-101 mutations are not capable of bypassing the complete lack of either the inductive signal, let-23, or let-60 ras, suggesting that an unc-101 mutation (and therefore the removal of one pathway of negative regulation) is not sufficient to promote vulval fates in the absence of inductive signal. The enhancement of the let-23(n1045) allele is difficult to interpret because the n1045 mutation results in a variety of abnormal mRNAs (Aroian et al. 1993b). We suspect that some species of proteins produced might have an inhibitory negative effect on the signal transduction (see Aroian and Sternberg 1991) and that this effect is enhanced by the lack of negative regulation by unc-101. A defect in unc-101, in combination with a mutation in the sli-1 locus, results in excessive vulval differentiation /G. Jongeward and P. Sternberg, in prep.|, supporting our hypothesis that unc-101 normally prevents excessive vulval differentiation.

\section{Roles of clathrin-associated complexes and unc-101}

AP complexes are thought to drive clathrin coat formation and to couple the clathrin lattice with distinct membrane proteins (Keen 1990; Pearse and Robinson 1990; Schmid 1992). The core of the complex (Fig. 1B), which contains amino-terminal halves of the large chains as well as the medium and small chains, can bind to clathrin trimers (Matsui and Kirchhausen 1990; Peeler et al. 1993). The function of each component of the AP complex is not well understood. It is possible that the medium chains function as regulators of the clathrinassociated protein complexes.

Because unc-101 mutations have many different effects on the behavior and development of C. elegans, the wild-type UNC-101 protein has important functions in many different types of cells. How would UNC-101 function in these cells? Besides a general role in regulating membrane trafficking, one function could be negative regulation of receptor-mediated signal transduction. 


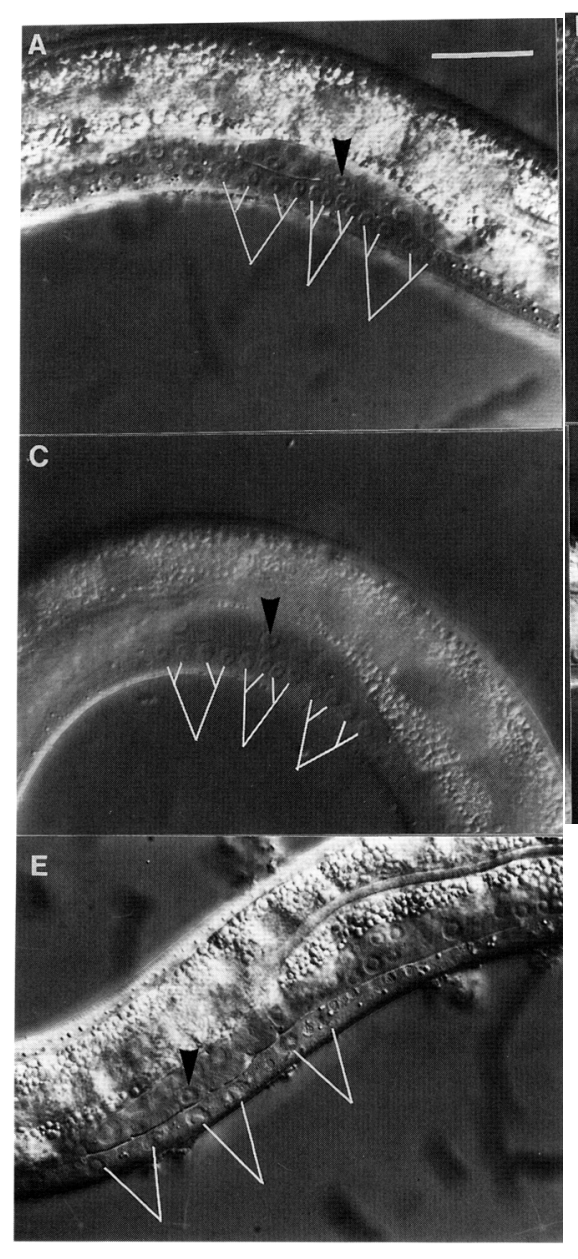

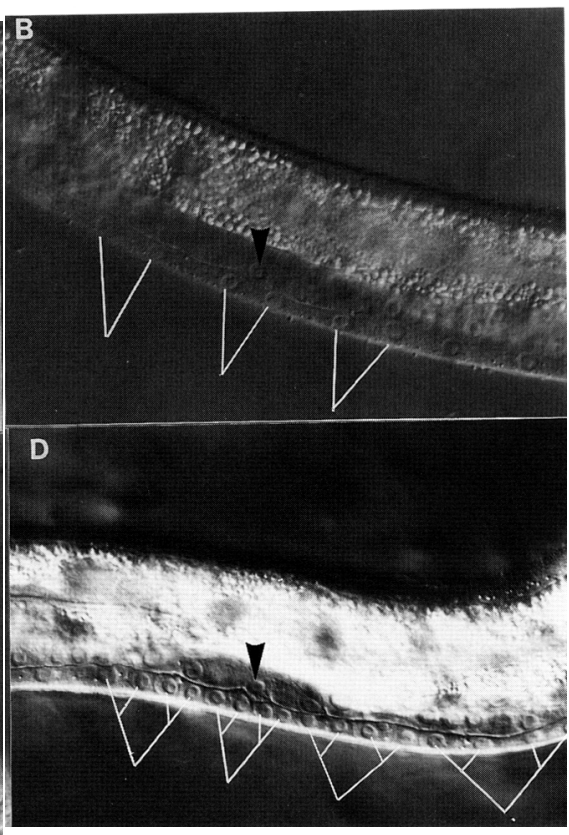

Figure 7. Rescue of suppression of the Vul phenotype of let-23(sy1) by the mouse/ nematode hybrid gene. The VPCs in the L3 molt stage, when the VPCs should have divided twice to generate four progeny, are shown. The triangles represent the anchor cell. (A) N2, three VPCs each generated four progeny; $(B)$ let-23(sy1), no VPC divided to generate four progeny; $(C)$ unc-101(sy108), same as N2; (D) unc-101(sy108); let23(sy1), four VPCs divided to generate four progeny; (E) unc-101(sy108);let23(sy1);Ex[AP47 hybrid], no VPC divided to generate four progeny. Bar, $20 \mu \mathrm{m}$.
Further genetic analysis may help to reveal the full spectrum of unc-101 interactions with other receptors.

On the basis of results from the biochemical studies on coated vesicles and assuming that UNC-101 is a trans-Golgi clathrin-associated protein, there are many possible molecular mechanisms by which UNC-101 could act within the cells involved in the C. elegans vulval induction. UNC-101 might regulate secretion of the LIN-3 signal within the anchor cell: UNC-101 might be involved in negative regulation of LIN-3 signal production by sequestering LIN-3 proteins from the secretion route after their synthesis. We do not favor this model because unc-101 mutants in a let-23(+) background do not have any excessive vulval differentiation while overexpression of lin-3 causes excessive vulval differentiation (Hill and Sternberg 1992). Another possible role of UNC-101 is regulation of transport of LET-23 receptors after their biosynthesis: UNC-101 might regulate LET-23 receptors by maintaining an intracellular pool of the receptors after their synthesis. This action could provide a post-translational regulation of protein activity. Hence, in the absence of an intracellular pool of the receptors, more LET-23 receptors whose activity is reduced by a reduction-of-function mutation could reach the cell surface, being capable of transducing more sig- nal. Sorkin and Carpenter (1993) have recently shown that $\alpha$-adaptins of the plasma membrane (AP-2 complex) interact with EGF receptor in A431 cells; AP-1 complexes containing UNC-101/AP47 might play an analogous role at other stages in intracellular trafficking. A third possible role of UNC-101 is attenuation of signal transduction after activation of the molecules in the pathway by directing intracellular degradation: One of the best known functions of the coated vesicles on the trans-Golgi is sorting of the lysosomal enzymes. This is accomplished by sorting mannose-6-phosphate receptors, which recognize lysosomal enzymes tagged with mannose-6-phosphate, and transferring these receptor/ ligand complexes to the lysosome. UNC-101 might be involved indirectly in the down-regulation of signal transduction components through controlling lysosomal or prelysosomal compartments; for example, in the absence of normal compartments, receptor might be recycled inappropriately.

\section{Materials and methods}

Strains and genetic methods

Methods for culturing, handling, and genetics of C. elegans were described by Brenner (1974). All genetic experiments unless oth- 
erwise indicated were performed at $20^{\circ} \mathrm{C}$. Mutagenesis protocol using TMP was provided by $M$. Yandell and L. Edgar (pers. comm.). The standard strain N2 and dpy-5(e61) were from Brenner (1974). The alleles for examining genetic interactions of unc-101 were as follows: lin-3: e1417, n378 (Horvitz and Sulston 1980); let-23: sy1, sy97, sy12 (Aroian and Sternberg 1991), $n 1045$ (Ferguson and Horvitz 1985); lin-2: $n 768$ (Ferguson and Horvitz 1985), e1309 (Horvitz and Sulston 1980); lin-7: e1413 (Ferguson and Horvitz 1985); lin-10: e1439 (Ferguson and Horvitz 1985); let-60: sy100dn (Han et al. 1990); lin-45: sy96 (Han et al. 1993). The starting strain for identifying the polymorphisms associated with unc-101, MT3618 [unc-75(e950) ced1(n1506) unc-59(e261)], which has a portion of chromosome I (between unc-75 and unc-59) from the TR679 mutator strain, was provided by S. Glass, T. Gerber, and R. Horvitz (pers. comm.). The unc-101 alleles sequenced were $m 1$ (D. Riddle, pers. comm.), rh6 (E. Hedgecock, pers. comm.), sy108, sy161 (G. Jongeward and P. Sternberg, in prep.), sy241 (this study), and sy237 (J. Lee, unpubl.).

\section{Isolation of new alleles of unc-101}

sy216 was isolated among the progeny of TMP-mutagenized ced-1 males mated with unc-101(rh6); dpy-5 hermaphrodites. A rare Unc-101 non-Dpy hermaphrodite was obtained after screening 11,000 gametes. A similar screen of 15,000 gametes using EMS was carried out, and two alleles, sy168 and sy169, were recovered. Sequencing these alleles indicated that these are the same mutation as sy108, presumably the result of gene conversion or the recovery of the maternal allele.

sy241 was isolated from a screen of 20,000 progeny of EMSmutagenized unc-101(sy108) +eDf24/hIn1/+unc-54(h1040)+] heterozygotes. eDf24 is a deficiency that deletes the rRNA genes on chromosome I, conferring lethality to the homozygotes for this deficiency. hIn1 is an inversion of chromosome I that suppresses recombination in the region between unc-75 and unc-54; one version of this balancer is marked with unc-54, facilitating the discrimination of the homozygotes. The heterozygous self-progeny of mutagenized parents were screened for rare Unc-101 animals, which would be unc-101(sy108) +eDf24/ hIn1[sy241 unc-54(h1040)+].

\section{Correlating genetic and physical maps}

The source of the syP6 and syP7 RFLPs was the strain MT3618 (unc-75 ced-1 unc-59), which contains a transposon-rich region between unc-75 and unc-59 (S. Glass, T. Gerber and R. Horvitz, pers. comm.), relative to the unc-101(sy108) strain, a typical Bristol strain. We picked 77 Unc-59 non-Unc- 75 recombinants from heterozygous progeny of ++ sy108+/unc-75 ced$1+$ unc-59 and selected animals homozygous for the recombinant chromosome. We digested genomic DNAs of two of these recombinants with HindIII, diluted, and self-ligated them, then used them as templates for PCR with Tc1 internal primers of Hill and Sternberg (1992), performing the inverse PCR as described by Ochman et al. (1988). We identified two polymorphisms, syP6 and $s y P 7,1.1 \mathrm{~kb}$ and $0.8 \mathrm{~kb}$ long, respectively, on an analytic agarose gel. We found that SyP6 was present in the congenic strain but was not present either in the +++ unc-59 or the ++ unc-101 unc-59 recombinants, indicating that this polymorphism is to the left of unc-101 on the genetic map. syP7 was present in ++ +unc-59, but not in + +unc-101 unc-59 recombinants; therefore, $s y P 7$ is near unc-101 and to the right of syP6. We used the flanking sequences of $s y P 6$ and $s y P 7$ as probe for physical and/or genetic mapping (Williams et al. 1992). To genetically map syP7 more accurately, we analyzed the remain- ing 75 recombinants and performed either Southern hybridization with $s y P 7$ as probe or PCR with syP7 primers. For PCR, two primers were made from the region flanking the $s y P 7$ polymorphism. EPRl， 5'-GGTGATAGCACCATATGGTTCC-3'; EPR2, 5'-ATATA GTGCTGTGCGGAACTC-3'. We designed these primers so that if the recombinants had the polymorphic transposon, the PCR-amplified band using either of these primers and the Tc1 internal primer would be $125 \mathrm{bp}$; long, and if not, an 80-bp band would be amplified from EPR 1 and EPR 2 as extending primers.

By hybridization to a YAC grid filter (Coulson et al. 1991), we located syP6 on the physical map on the right arm of chromosome I. syP7 was located $\sim 600 \mathrm{~kb}$ to the right of $s y P 6$ on the same set of overlapping genomic clones. Using 38 of the recombinants for which we scored the ced-1 marker, 5 were between unc-75 and ced-1, 12 were between ced-1 and unc-101, and 21 were between unc-101 and unc-59.

\section{Cosmids and $\mathrm{C}$. elegans physical map}

We obtained all cosmids and the physical map data from A. Coulson and J. Sulston (Medical Research Council, Cambridge, UK; Coulson et al. 1986, 1988).

\section{DNA-mediated transformation of unc-101 mutants}

Microinjection of cosmids or subcloned DNAs was described by Mello et al. (1991). We used unc-101(sy108) animals as host strain for rescuing the Unc and the lethality of the visible allele. We coinjected unc-101(sy108) animals with pRF4, which bears a dominant rol-6 mutation that results in a rolling phenotype. Selection of this marker phenotype facilitates selecting the transgenic animals. We used unc-101(sy108);1et-23(sy1) animals to examine the rescue of the suppression of the let-23(sy1) Vul phenotype. We injected these animals with the rescuing subclone pIL5 without any other marker, because the rescue of the Unc phenotype itself serves as a good marker for the presence of the transgene.

\section{DNA manipulations, sequencing, and analysis}

All procedures for handling genomic DNA and cDNA were as described (Sambrook et al. 1989). The Macvector software package of IBI (New Haven CT.) and the software of the Genetics Computer Group (Program manual for the GCG package, v. 7, 1991, University of Wisconsin, Madison) was used to edit the genomic and cDNA sequences. Data base search was performed using the BLAST program. Pileup and Prettyplot commands were used to generate the comparison of the amino acid sequences.

\section{Sequence of mutant alleles}

We made 10 PCR primers from the intron sequences, 5 ' end and $3^{\prime}$-nontranslated region as follows: INT1, TTCCGCTAATTTTCTCCGG; INT2, ATTGCGTCATTTTCAACGG; INT3, CGCTCCAATGATAAAACAC; INT4, GCATTTTCGCATTGGAGCG; INT5, AAATGTGTTTTTCGACTCG; INT6, AAAAACTAGGCCACATCAC; INT7, AAGTCAGGCCATGCCTCAA; INT8, CATAAATCTCACATTGGGCA; INT9, GAGAATTATGTGATTTTTTG; and INT10, CTCGGCCACGGTCGTTTTT. With these PCR primers, we could amplify all the exons and their flanking intron sequences. To amplify by PCR the exons and their boundaries, we used genomic DNA or single worms of the homozygous mutant. We directly se- 
quenced the amplified DNAs using the same sets of primers (Kretz et al. 1989).

\section{Construction of hybrid genes}

To construct an unc-101/AP47 hybrid gene, we used the unc101 rescuing plasmid pJL2, the unc-101 cDNA subclone, and the mouse AP47 cDNA subclone as sources for the sequences. We kept the first two introns of the rescuing pIL2 plasmid in this construction because plL 2 has only 95 nucleotides 5 to the SL-1 acceptor sequence and because expression of transgenes is more efficient in the presence of introns (e.g., Brinster et al. 1988; Buchman and Berg 1988; Fire et al. 1990). The rescuing plasmid pJL2 was digested with NruI and partially with EcoRV, and the 7.2-kb fragment was purified from a low melting point agarose gel and ligated with the 0.9-kb NruI-EcoRV fragment from the unc-101 cDNA subclone. The resulting plasmid was the unc-101 cDNA hybrid gene. We made two PCR primers from the AP47 cDNA sequence to amplify the corresponding region of the NruI-EcoRV fragment of unc-101 cDNA: 47-1, 5'-CGACAACTTTGTCATCATCTA; 47-2, 5'-ATCCACTCTTCTCAATGATTTTC- $3^{\prime}$. To facilitate subcloning, we replaced three most $5^{\prime}$ nucleotides of $47-1$ and $47-2$ primers with the recognition sequence of $N r u I$ and $E c o$ RV, respectively. This replacement does not change the encoded amino acid. We ligated the amplified DNA into the pJL2 NruI-EcoRV fragment. We confirmed the correct reading frame by regeneration of the NruI and EcoRV sites. This construct has the 5' region of unc-101, including the $5^{\prime}$ region of the rescuing plasmid pJL2 and the coding region with two introns up to cDNA nucleotide 388 , fused in-frame to the AP47 cDNA from 389 to 1281 , unc-101 cDNA nucleotide from 1282 to the stop codon, and all of the 3 '-untranslated region of unc-101. The control construct has a deletion of the NruI-EcoRV fragment (equivalent to nucleotides 389-1281 of the cDNA).

Assay for the rescue of the phenotypes of unc-101 animals by the nematode/mouse hybrid gene

We obtained stable lines of transgenic animals after microinjection of the hybrid gene. For the Unc phenotype rescue, we transferred five transgenic animals to a spot on new plates; and after the given time, we photographed the tracks that the animals created by moving on the bacterial lawn viewed in a Wild M5A stereomicroscope. For the rescue of the suppression of let23(sy1) Vul phenotype, we examined the vulval induction of the transgenic animals bearing the hybrid gene in their late L3 stages using Nomarski optics.

\section{Analysis of sy216}

To phenotypically examine sy216 and other unc-101 alleles, we constructed trans-heterozygous animals bearing sy216/sy108. More sy216/sy108 heterozygous animals $(74 \%)$ are inviable than of sy108/sy108 animals (45\%). Suppression of the Vul phenotype of let-23(sy1) is not enhanced. sy216/sy108;let-23(sy1) animals averaged 3.6 VPCs undergoing vulval differentiation, which is the same level of vulval differentiation of sy108/ sy108; let-23(sy1) animals ( $n=20$ for both).

Because TMP is known to induce small deletions at high frequency (L. Edgar, pers. comm.), we suspected that sy216 could be a small deficiency that deletes neighboring genes as well as unc-101. To address this issue genetically, we tested whether any of the nearest genes are deleted by sy216. We constructed the trans-heterozygote unc-101(sy216)/eDf3. eDf3 fails to complement the mutations that define the nearest ge- netically defined loci to the right: unc-59, let-201, let-202, and let-203. This heterozygote is viable and wild type. Therefore, sy216 does not delete the nearest genetically defined loci to the right. sy216 does not delete the nearest known gene to the left, ced-1, because a sy216 chromosome can donate a wild-type copy of ced-1 in a recombination event. Specifically, an animal of genotype $d p y-5+s y 216 / h \operatorname{In} 1$ was found among the crossprogeny of a +ced-1 sy216/dpy-5+unc-101(rh6) hermaphrodite mated with hIn1 males. However, it is likely that there are essential genes in the interval between ced-1 and $e D f 3$, as this region is not saturated for lethal mutations.

To examine the rescue of the lethality of sy216 by the rescuing cosmid W05A3, we used sy216/hIn1 unc-54(h1040) heterozygotes as host for the transgene. $h \operatorname{In} 1$ is an inversion that suppresses recombination in the region between unc-75 and unc-59 (Zetka and Rose 1992). We coinjected the W05A3 cosmid and the pRF4 marker DNAs, picked rolling F1 transgenic animals, transferred $20 \mathrm{~F} 2$ individual animals to new plates, and checked their segregation of unc-54 marker. All 20 segregated unc-54, indicating that there was no animal of genotype sy216/sy216 carrying a W05A3 transgene.

To amplify genomic DNA from the sy216/sy216-arrested animals, we picked two or three arrested animals and performed PCR as described above. As an internal positive control, we used a set of let-23 PCR primers in the same PCR reactions with unc-101 primers. We tried five different sets of unc-101 primers but only recovered an amplified band of let-23 genomic DNA from the let-23 primers.

To detect any polymorphisms linked to sy216, we used genomic DNA of sy216/hIn1 heterozygotes digested with various restriction enzymes in a Southern hybridization with W05A3 cosmid as probe. We did not detect any polymorphism compared with control hIn1 homozygotes.

\section{Acknowledgments}

We are grateful to D. Riddle and E. Hedgecock for sharing their unc-101 alleles. We thank S. Lemmon and G. Payne for sharing their unpublished results. We thank L. Edgar for the TMP mutagenesis protocol; T. Kirchhausen for the AP47 cDNA; and A. van der Bliek, T. Clandinin, H. Chamberlin, R. Hill, A. Golden, L. Huang, P. Kayne, J. Liu, T. Kirchhausen, and R. Palmer for their comments. We also thank A. Coulson and the MRC and St. Louis group for all the cosmids, physical maps, and YACs; S. Glass, T. Gerber, and R. Horvitz for providing the MT3618 strain; M. Zetka and A. Rose for providing the hIn1 strain; and I.-Y. Ho and A. Rose for communicating their data on sy216. Some nematode strains were provided by Caenorhabditis elegans Genetic Toolkit Project, which is funded by the National Institutes of Health National Center for Research Resources. P.W.S. is an investigator of the Howard Hughes Medical Institute. This research has been supported by U.S. Public Health Service grant HD 23690 to P.W.S.

The publication costs of this article were defrayed in part by payment of page charges. This article must therefore be hereby marked "advertisement" in accordance with 18 USC section 1734 solely to indicate this fact.

\section{References}

Ahle, S., A. Mann, U. Eichelsbacher, and E. Ungewickell. 1988. Structural relationships between clathrin assembly proteins from the Golgi and the plasma membrane. EMBO /. 7: 919929.

Aroian, R.V. and P.W. Sternberg. 1991. Multiple functions of 
let-23, a C. elegans receptor tyrosine kinase gene required for vulval induction. Genetics 128: 251-267.

Aroian, R.V., M. Koga, J.E. Mendel, Y. Ohshima, and P.W. Sternberg. 1990. The let-23 gene necessary for Caenorhabditis elegans vulval induction encodes a tyrosine kinase of the EGF receptor subfamily. Nature 348: 693-699.

Aroian, R.V., G.M. Lesa, and P.W. Sternberg. 1993a. Mutations in the Caenorhabditis elegans let-23 EGF receptor-like gene define elements important for cell-type specificity and function. $E M B O J$. 12(13): (in press).

Aroian, R.V., A.D. Levy, M. Koga, Y. Ohshima, J.M. Kramer, and P.W. Sternberg. 1993b. Splicing in Caenorhabditis elegans does not require an AG at the $3^{\prime}$ splice acceptor site. Mol. Cell. Biol. 13: 626-637.

Barstead, R.J. and R.H. Waterston. 1989. The basal component of the nematode dense-body is vinculin. I. Biol. Chem. 264: 10177-10185.

Bazinet, C., A.L. Katzen, M. Morgan, A.P. Mahowald, and S.K. Lemmon. 1993. The Drosophila clathrin heavy chain gene: Clathrin function is essential in a multicellular organism. Genetics 134: 1119-1134.

Brenner, S. 1974. The genetics of Caenorhabditis elegans. Genetics 77: 71-94.

Brinster, R.L., J.M. Allen, R.R. Behringer, R.E. Gelinas, and R.D. Palmiter. 1988. Introns increase transcriptional efficiency in transgenic mice. Proc. Natl. Acad. Sci. 85: 836-840.

Brodsky, F.M. 1988. Living with clathrin: Its role in intracellular membrane traffic. Science 242: 1396-1402.

Buchman, A.R. and P. Berg. 1988. Comparison of intron-dependent and intron-independent gene expression. Mol. Cell. Biol. 8: 4395-4405.

Coulson, A.R., J. Sulston, S. Brenner, and J. Karn. 1986. Toward a physical map of the genome of the nematode Caenorhabditis elegans. Proc. Natl. Acad. Sci. 83: 7821-7825.

Coulson, A., R. Waterston, J. Kiff, J. Sulston, and Y. Kohara. 1988. Genome linking with yeast artificial chromosomes. Nature 335: 184-186.

Coulson, A., Y. Kozono, B. Lutterbach, R. Shownkeen, J. Sulston, and R. Waterson. 1991. YACs and the C. elegans genome. BioEssays 13: 413-417.

Ferguson, E. and H.R. Horvitz. 1985. Identification and characterization of 22 genes that affect the vulval cell lineages of Caenorhabditis elegans. Genetics 110: 17-72.

Fire, A., S. White-Harrison, and D. Dixon. 1990. A modular set of $l a c Z$ fusion vectors for studying gene expression in Caenorhabditis elegans. Gene 93: 189-198.

Han, M., R. Aroian, and P.W. Sternberg. 1990. The let-60 locus controls the switch between vulval and non-vulval cell types in C. elegans. Genetics 126: 899-913.

Han, M., A. Golden, Y. Han, and P.W. Sternberg. 1993. C. elegans lin-45 raf gene participates in let-60 ras stimulated vulval differentiation. Nature 363: 133-140.

Hedgecock, E.M., J.G. Culotti, J.N. Thomson, and L.A. Perkins. 1985. Axonal guidance mutants of Caenorhabditis elegans identified by filling sensory neurons with fluorescein dyes. Dev. Biol. 111: 1153-1157.

Hill, R.J. and P.W. Stemberg. 1992. The lin-3 gene encodes an inductive signal for vulval development in C. elegans. $\mathrm{Na}$ ture 358: $470-476$.

Horvitz, H.R. and P.W. Sternberg. 1991. Multiple intercellular signalling systems control the development of the C. elegans vulva. Nature 351: 535-541.

Horvitz, H.R. and J.E. Sulston. 1980. Isolation and genetic characterization of cell-lineage mutants of the nematode Caenorhabditis elegans. Genetics 96: 435-454.

Keen, J.H. 1987. Clathrin assembly proteins: Affinity purifica- tion and a model for coat assembly. $J$. Cell Biol. 105: 19891998.

1990. Clathrin and associated assembly and disassembly proteins. Annu. Rev. Biochem. 59: 415-438.

Keen, J.H., M.H. Chesnut, and K.A. Beck. 1987. The clathrin coat assembly polypeptide complex: Autophosphorylation and assembly activities. J. Biol. Chem. 262: 12182-12188.

Kirchhausen, T., K.L. Nathanson, W. Matsui, A. Vaisberg, and E.P. Chow. 1989. Structural and functional division into 2 domains of the large $(100-\mathrm{kD}$ to $115-\mathrm{kD})$ chains of the clathrin-associated protein complex AP-2. Proc. Natl. Acad. Sci. 86: 2612-2616.

Kirchhausen, T., A.C. Davis, S. Frucht, B.O. Greco, and G.S. Payne. 1991. AP17 and AP19, the mammalian small chains of the clathrin-associated protein complexes show homology to YAP17p, their putative homolog in yeast. $J$. Biol. Chem. 266: 1153-1157.

Krause, M. and D. Hirsh. 1987. A trans-spliced leader sequence on actin mRNA in Caenorhabditis elegans. Cell 49: 753761.

Kretz, K.A., G.S. Carson, and J.S. O'Brien. 1989. Direct sequencing from low-melting agarose with sequenase. Nucleic Acids Res. 17: 5864.

Matsui, W. and T. Kirchhausen. 1990. Stabilization of clathrin coats by the core of the clathrin-associated protein complex AP-2. Biochemistry 29: 10791-10798.

Mello, C.C., J.M. Kramer, D. Stinchcomb, and V. Ambros. 1991. Efficient gene transfer in C. elegans after microinjection of DNA into germline cytoplasm: Extrachromosomal maintenance and integration of transforming sequences. $E M B O F$. 10: $3959-3970$.

Nakayama, Y., M. Goebl, B.O. Greco, and S. Lemmon, E.P.C. Chow, and T. Kirchhausen. 1991. The medium chains of the mammalian clathrin-associated proteins have a homolog in yeast. Eur. J. Biochem. 202: 569-574.

Ochman, H., A.S. Gerber, and D.L. Hartl. 1988. Genetic applications of an inverse polymerase chain reaction. Genetics 120: $621-623$.

Pearse, B.M.F. and M.S. Robinson. 1990. Clathrin, adapters, and sorting. Annu. Rev. Cell Biol. 6: 151-171.

Peeler, J.S., W.C. Donzell, and R.G.W. Anderson. 1993 The appendage domain of the AP-2 subunit is not required for assembly or invagination of clathrin-coated pits. J. Cell. Biol. 120: 47-54.

Ruvkun, G., V. Ambros, A. Coulson, R. Waterston, J. Sulston, and H.R. Horvitz. 1989. Molecular genetics of the Caenorhabditis elegans heterochronic gene lin-14. Genetics 121: 501-516.

Sambrook, J., E.F. Fritsch, and T. Maniatis. 1989. Molecular cloning: A laboratory manual, 2nd ed. Cold Spring Harbor Laboratory Press, Cold Spring Harbor, New York.

Seeger, M. and G.S. Payne. 1992. Selective and immediate effects of clathrin heavy-chain mutations on Golgi membraneprotein retention in Saccharomyces cerevisiae. I. Cell. Biol. 118: $531-540$.

Schmid, S.L. 1992. The mechanism of receptor-mediated endocytosis-More questions than answers. BioEssays 14: $589-596$.

Sorkin, A., and G. Carpenter. 1993. Interaction of activated EGF receptors with coated pit adaptins. Science 261: 612-615

Sternberg, P. W. 1993. Intercellular signaling and signal transduction in C. elegans. Annu. Rev. Genet. 27: 497-520.

Thomas, J.H. 1990. Genetic analysis of defecation in Caenorhabditis elegans. Genetics 124: 855-872.

Thurieau, C., J. Brosius, C. Burne, P. Jolles, J. Keen, R.J. Mattaliano, E.P. Chow, K. Ramachandran, and T. Kirchhausen. 
1988. Molecular cloning of complete amino acid sequence of AP50, an assembly protein associated with clathrin-coated vesicles. DNA 7: 663-669.

Waterston, R., C. Martin, M. Craxton, C. Huynh, A. Coulson, L. Hillier, R. Durbin, P. Green, R. Shownkeen, N. Halloran, M. Metzstein, T. Hawkins, R. Wilson, M. Berks, Z. Du, K. Thomas, J. Thierry-Mieg, and J. Sulston. 1992. A survey of expressed genes in Caenorhabditis elegans. Nature Genet. 1: 114-123.

Williams, B.D., B. Schrank, C. Huynh, R. Shownkeen, and R.H. Waterston. 1992. A genetic mapping system in Caenorhabditis elegans based on polymorphic sequence-tagged sites. Genetics 131: 609-624.

Zetka, M.C. and A.M. Rose. 1992. The meiotic behavior of an inversion in Caenorhabditis elegans. Genetics 131: 321332. 


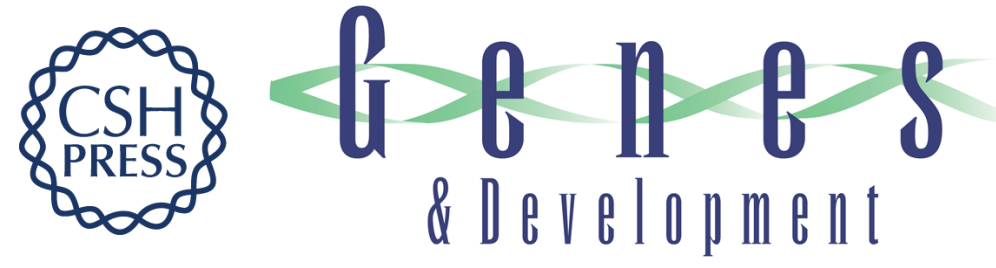

\section{unc-101, a gene required for many aspects of Caenorhabditis elegans development and behavior, encodes a clathrin-associated protein.}

J Lee, G D Jongeward and P W Sternberg

Genes Dev. 1994, 8:

Access the most recent version at doi:10.1101/gad.8.1.60

References

License

Email Alerting Service
This article cites 43 articles, 23 of which can be accessed free at:

http://genesdev.cshlp.org/content/8/1/60.full.html\#ref-list-1

Receive free email alerts when new articles cite this article - sign up in the box at the top right corner of the article or click here.

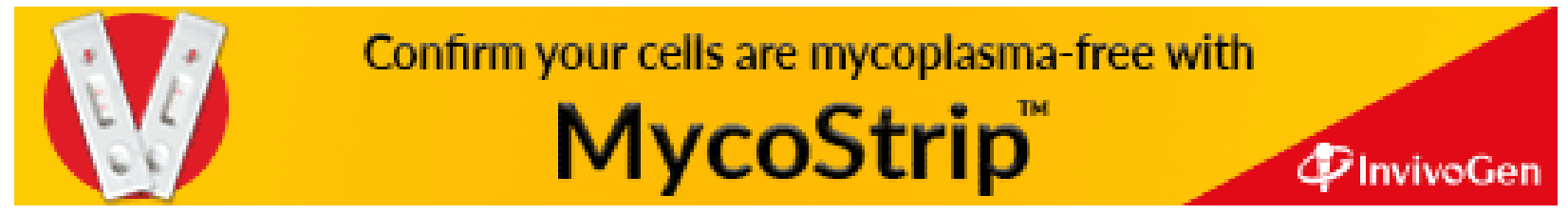

